\title{
The Use of Handheld Ultrasound in Early Diagnosis and Treatment of Life Threatening Cardiac Condition: A Case Report of Early Diagnosis and Treatment of Cardiac Tamponade
}

\begin{abstract}
Ultraportable handheld ultrasound machines have been available in the health care market since 2010. There are wide array of uses for handheld ultrasound including applications in the field of obstetrics, emergency medicine, and cardiovascular imaging. Handheld ultrasound helps clinicians perform pointof-care bedside assessment and to make timely decisions which impact the outcome of patients. We present a case report demonstrating the use of handheld ultrasound helped with early diagnosis and treatment of cardiac tamponade.
\end{abstract}

\section{Introduction}

A handheld ultrasound (HHU) is defined as a "pocket-size, hand-held imaging devices." [1]. The European Association of Echocardiography [1] gave eight recommendations for its use: 1 . Complement to a physical examination in the coronary and intensive care unit, 2. Tool for a fast initial screening in an emergency setting, 3. Cardiologic counselling in or outside health-care facilities and hospitals, 4. First cardiac evaluation in ambulances, 5. Screening programs in schools, industry, and community activities, 6. Triaging candidates for a complete echocardiographic examination, 7. Teaching tool, 8. Semiquantification of extravascular lung water. In literature, there are myriad uses of HHU that have been described including epidural analgesia for women in labor [2], evaluation of breast mass $[3,4]$, evaluation of blood supply prior to plastic surgery [5], measurement of optic nerve sheath diameter to evaluate for intracranial pressure [6], detection of fetal growth restriction [7], evaluation of trauma patients [8], diagnosis of bone fracture [9], teaching cardiovascular anatomy to medical students [10], and evaluation for heart failure [11].

\section{Case Report}

A 34 year-old male with history of hypertension, diabetes, and end stage renal disease on hemodialysis who was sent to the emergency room due to hypotension during outpatient hemodialysis. In the emergency room, patient was hemodynamically unstable. Patient was intubated for acute respiratory failure and was started on pressors due to shock. Patient's cardiac exam showed diminished heart sounds and bedside echo performed by a HHU (GE Vscan®) showed a large pericardial effusion (Figure 1a). Other physical exam findings included pulsus paradoxus which confirmed cardiac tamponade. An emergent pericardiocentesis was performed at bedside, with the guidance of $\mathrm{HHU}$, and $2.5 \mathrm{~L}$ of pericardial effusion was removed. Repeat bedside echo showed trace residual pericardial

Case Report
Volume 9 Issue 6 - 2017
Howard Lan* and Nirav Mamdlani
Loma Linda University Medical Center, USA
*Corresponding author: Howard Lan, Loma Linda
University Medical Center, Loma Linda, CA, USA,
Email: HWLan@llu.edu
Received: September 26, 2017 | Published: October 06,
2017

effusion with expanded right ventricle filling (Figure $1 \mathrm{~b}$ ), and patient's hemodynamics improved immediately.

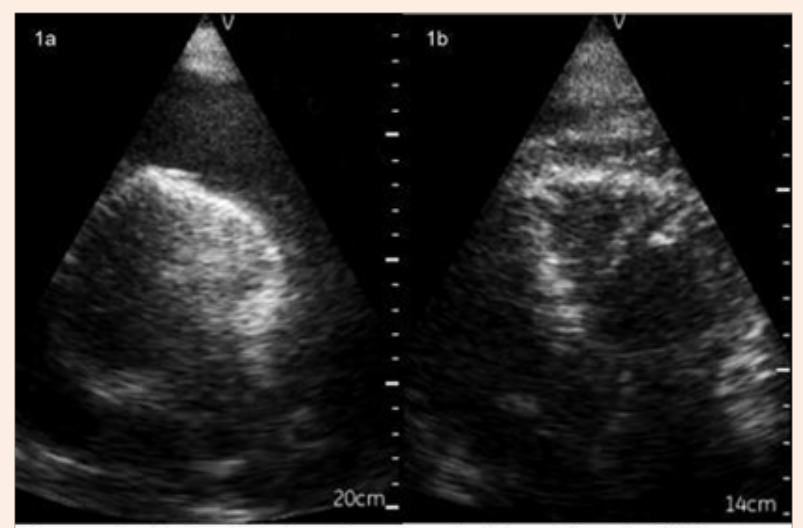

Figure 1:

a) Apical 4 chamber view in mayo format (RV on right side) showing large pericardial effusion and RV diastolic collapse.

b) Post 2.5L pericardiocentesis showing residual small size pericardial effusion with improved RV filling. Images were downlosded from GE $\operatorname{Vscan}^{\mathrm{TM}}$ (Fairfield CT) handheld ultrasound.

\section{Discussion}

With advancing technology in ultrasound, devices are now smaller, more portable, cheaper, and have improved interface with electronic medical record [12]. Utilization of HHU for daily use in clinical practice might become universal over time. There 
is much debate on whether HHU will replace the stethoscope $[13,14]$, however, most would agree that HHU augments physical findings at bedside [15] and could assist physicians in making important clinical decisions.

Transthoracic echocardiogram performed by HHU has been shown to be cost effective and could provide accurate detection of cardiac structural and functional abnormalities [16]. When used by cardiologists, HHU provides a more accurate diagnosis than physical examination alone for the majority of common cardiovascular abnormalities [17]. In this case report, the use of HHU to supplement the cardiac exam resulted in early diagnosis and treatment of cardiac tamponade, a medical emergency. In literature, there are two prior reports of cardiac tamponade that were diagnosed and treated with HHU guidance $[18,19]$, both with good clinical outcome similar to our case.

\section{Conclusion}

There are variety of uses for HHU in the field of medicine. HHU is currently used as an adjunct to the standard physical examination at bedside. In this case report, the use of HHU supplementing the cardiac exam lead to early diagnosis and treatment of cardiac tamponade which resulted in immediate improvement in patient's hemodynamics and outcome.

\section{Conflict of Interest}

None

\section{References}

1. Tofield A (2011) The use of pocket size imaging devices: a position statement by the European Association of Echocardiography. Eur Heart J 32(4): 385-386

2. Seligman KM, Weiniger CF, Carvalho B (2017) The Accuracy of a Handheld Ultrasound Device for Neuraxial Depth and Landmark Assessment: A Prospective Cohort Trial. Anesth Analg, doi:10.1213/ ANE.0000000000002407.

3. Barr RG, DeVita R, Destounis S, Manzoni F, De Silvestri A, et al. (2017) Agreement Between an Automated Volume Breast Scanner and Handheld Ultrasound for Diagnostic Breast Examinations. J Ultrasound Med 36(10): 2087-2092.

4. Schmachtenberg C, Fischer T, Hamm B, Bick U (2017) Diagnostic Performance of Automated Breast Volume Scanning (ABVS) Compared to Handheld Ultrasonography With Breast MRI as the Gold Standard. Acad Radiol 24(8): 954-961.

5. Lethaus B, Loberg C, Kloss-Brandstätter A, Bartella AK, Steiner T, et al. (2017) Color duplex ultrasonography versus handheld Doppler to plan anterior lateral thigh flaps. Microsurgery 37(5): 388-393.
6. Johnson GG, Zeiler FA, Unger B, Hansen G, Karakitsos D, et al. (2016) Estimating the accuracy of optic nerve sheath diameter measurement using a pocket-sized, handheld ultrasound on a simulation model. Crit Ultrasound J 8(1): 18.

7. Haragan AF, Hulsey TC, Hawk AF, Newman RB, Chang EY (2015) Diagnostic accuracy of fundal height and handheld ultrasoundmeasured abdominal circumference to screen for fetal growth abnormalities. Am J Obstet Gynecol 212(6): 820.e1-8.

8. Baugher KM, Euerle BD, Sommerkamp SK, Witting MD (2014) Image quality evaluation of a portable handheld ultrasound machine for the focused assessment with sonography for trauma examination. Am J Emerg Med 32(4): 389-391.

9. Brooks AJ, Price V, Simms M, Ward N, Hand CJ (2004) Handheld ultrasound diagnosis of extremity fractures. J R Army Med Corps 150(2): 78-80.

10. Wittich CM, Montgomery SC, Neben MA, Palmer BA, Callahan MJ, et al. (2002) Teaching cardiovascular anatomy to medical students by using a handheld ultrasound device. JAMA 288(9): 1062-1063.

11. Hebert K, Horswell R, Heidenreich P, Miranda J, Arcement L (2010) Handheld ultrasound, B-natriuretic peptide for screening stage B heart failure. South Med J 103(7): 616-622.

12. https://www.lumify.philips.com/web/

13. Fredricks T (2016) Point-of-care ultrasound: It's no replacement for the stethoscope. J Fam Pract 65(10): 734.

14. Copetti R (2016) Is lung ultrasound the stethoscope of the new millennium? Definitely yes! Acta Med Acad 45(1): 80-81.

15. Kimura BJ (2017) Point-of-care cardiac ultrasound techniques in the physical examination: better at the bedside. Heart 103(13): 987-994.

16. Kitada R, Fukuda S, Watanabe H, Oe H, Abe Y, et al. (2013) Diagnostic accuracy and cost-effectiveness of a pocket-sized transthoracic echocardiographic imaging device. Clin Cardiol 36(10): 603-610.

17. Mehta M, Jacobson T, Peters D, Le E, Chadderdon S, et al. (2014) Handheld ultrasound versus physical examination in patients referred for transthoracic echocardiography for a suspected cardiac condition. JACC Cardiovasc Imaging 7(10): 983-990.

18. Byhahn C, Bingold TM, Zwissler B, Maier M, Walcher F (2008) Prehospital ultrasound detects pericardial tamponade in a pregnant victim of stabbing assault. Resuscitation 76(1): 146-148.

19. Otsuka H, Sato T, Morita S, Nakagawa Y, Inokuchi S (2016) A Case of Blunt Traumatic Cardiac Tamponade Successfully Treated by Outof-hospital Pericardial Drainage in a Doctor-helicopter Ambulance Staffed by Skilled Emergency Physicians. Tokai J Exp Clin Med 41(1): $1-3$. 